\title{
Impaired HLA capping capacity of peripheral blood lymphocytes in Duchenne muscular dystrophy
}

\author{
A SENSI*, ANNA VENTUROLI $\dagger$, SERENA TRANIELLO $\dagger$, M LUCC + , \\ C VUllo $\ddagger$, C CONIGHI*, P L MATTIUZ*, AND O R BARICORDI* \\ From * the Institute of Medical Genetics, and $\dagger$ the Institute of Biochemistry, University of Ferrara; \\ and $\ddagger$ the Pediatric Division, USL 31, Ferrara, Italy.
}

SUMMARY The cap capacity in nine Duchenne muscular dystrophy (DMD) patients and in $23 . \vec{\omega}$ healthy male subjects (14 adults and nine neonates) has been investigated by inducing capping of $\vec{\infty}$ HLA molecules. The evaluation of capping percentages ranged in healthy controls from 44 to 61 with a mean value of $53 \cdot 39 \pm 4 \cdot 89$, while DMD patients displayed cap capacity of percentages varying from 15 to 38 with a mean value of $31 \cdot 5 \pm 7 \cdot 42$. Statistical significance of the differences between the two groups, assessed by the Mann-Whitney $U$ test, was $p<0.00003$.

A correlation was found in DMD patients between cap capacity and age $(\tau=+0 \cdot 657, \mathrm{p}=0 \cdot 012)$. The results confirm previous reports of Ig capping impairment noted in B cells of the whole lymphocyte population, supporting the hypothesis of a systemic cellular defect in DMD patients. The data obtained suggest that HLA capping could overcome some of the technical difficulties of Ig capping and could be used as a diagnostic aid in antenatal detection of DMD.

Various cell membrane abnormalities affecting different cell populations, such as muscle cells, erythrocytes, T lymphocytes, and B lymphocytes, have been described in DMD by several ininvestigators. ${ }^{1-3}$

In spite of the fact that the basic defect is still unknown, a large number of studies have been performed to establish the usefulness of these membrane phenomena as a diagnostic test in antenatal diagnosis and carrier detection. ${ }^{1-10}$ One of the main functional defects in DMD cell membranes has been shown to be B lymphocyte Ig capping capacity. ${ }^{12}$ 4-10

According to the 'fluid mosaic theory' of cell membrane organisation proposed by Singer and Nicolson, 11 capping can be defined as an immunologically induced redistribution of cell membrane antigens. This phenomenon is controlled by a wide number of factors, such as membrane structure and composition, availability of energy in the form of ATP, structure and function of cell membrane associated contractile elements, and ionic intracellular concentrations with particular regard to $\mathrm{Ca}^{++}{ }_{12-15}$

Impairment of capping capacity of lymphocytes in DMD was first noted by Verrill et al, ${ }^{1}$ who

Received for publication 28 July 1983. Accepted for publication 24 October 1983. observed a reduction in lymphocyte capping induced by FITC anti-immunoglobulin sera in patients when compared to healthy controls.

Studies performed in patients with Becker, limb girdle, facioscapulohumeral, and congenital muscular dystrophies showed a similar diminished B cell capping capacity. ${ }^{2} 716$ These early results suggested that the membrane abnormality might be used in the diagnosis of muscular dystrophies. This exciting hypothesis led to a large number of studies on DMD patients and carriers, but conflicting results were obtained. 45 7-10

To achieve a better evaluation of the capping phenomenon in DMD patients, we extended the investigation to the whole lymphocyte population inducing the cap formation of the HLA molecular complex, which consists of membrane glycosylated peptide chains.

Since class I antigens are expressed on both $T:$ and $B$ lymphocyte membranes, the determination of capping capacity in a large number of cells by indirect immunofluorescence improves the results.

\section{Materials and methods}

SUBJECTS

Blood samples were obtained from nine DMD subjects. Patients were carefully selected using 
clinical, serum enzyme, and electromyographic criteria. Fourteen adult males, tested for CK levels and without any muscular disorder, served as healthy controls. Nine newborn males, without family history of muscular dystrophy, five of whom had high CK levels (table 1), were also tested for HLA capping. None of the neonates were affected on subsequent clinical evaluation and all the CK levels were within the normal range 2 weeks later.

\section{METHODS}

Lymphocyte studies were performed within 10 hours of the collection of the blood. Peripheral lymphocytes were isolated from heparinised venous blood by the standard Ficoll-Hypaque density gradient technique.

To a cell pellet containing $0.3 \times 10^{6}$ cells, $50 \mu \mathrm{l}$ of human polyspecific anti-HLA serum (TSRP 32) were added. The samples were incubated on ice for 30 minutes, washed three times in phosphate buffered saline (PBS), and resuspended in $50 \mu l$ of 1:10 FITC conjugated rabbit anti-human polyvalent immunoglobulins (MILES), previously dialysed overnight against PBS to remove the azide preservative. The lymphocytes were then put on ice for 30 minutes, washed three times in cold PBS, and incubated for 30 minutes at $37^{\circ} \mathrm{C}$. The fluorescein labelled cell suspension was placed on a cold slide, covered with a coverslip, and immediately observed under visible and fluorescent light with a Zeiss Photomicroscope III, equipped with the fluorescent RS III system and XBO lamp.

At least 100 fluorescent live cells, without endocytosed fluorescent material, were counted per

TABLE 1 Capping percentages, serum CK levels, and age in healthy controls.

\begin{tabular}{rlrc}
\hline No & Capping $(\%)$ & $C K(U / l)$ & Age \\
\hline 1 & 61 & 49 & 37 years \\
2 & 59 & 61 & 41 years \\
3 & 59 & 39 & 47 years \\
4 & 58 & 67 & 63 years \\
5 & 58 & 36 & 34 years \\
6 & 58 & 58 & 4 days \\
7 & 58 & 77 & 32 days \\
8 & 56 & 103 & 5 days \\
9 & 55 & 96 & 8 days \\
10 & 55 & 43 & 30 years \\
11 & 54 & 85 & 30 years \\
12 & 54 & 75 & 37 years \\
13 & 54 & 26 & 47 days \\
14 & 54 & 232 & 4 days \\
15 & 53 & 275 & 4 days \\
16 & 52 & 37 & 49 years \\
17 & 50 & 74 & 32 years \\
18 & 49 & 90 & 7 days \\
19 & 48 & 140 & 8 days \\
20 & 48 & 58 & 35 years \\
21 & 47 & 94 & 30 years \\
22 & 44 & 83 & 36 years \\
23 & 44 & 85 & 29 years \\
\hline
\end{tabular}

slide. Cells were regarded as having reached the capping stage when at least $75 \%$ of the surface fluorescence was confined to one hemisphere, as suggested by Fitzsimmons and co-workers. ${ }^{4}$ For every subject the capping capacity was expressed as a percentage of the total number of fluorescent cells.

The evaluation of serum creatine kinase (CK) was performed by the method reported by Rosalki ${ }^{17}$ and the enzyme activity was expressed as $U / 1$. Statistical evaluation of the significance between capping percentages in DMD and healthy controls was performed by the Mann-Whitney $U$ test, ${ }^{18}$ and the correlations of capping values with $\mathrm{CK}$ levels and ages examined using the non-parametric Kendall rank correlation coefficient. ${ }^{18}$ Significant correlations were further analysed by the Kendall partial rank correlation coefficient. ${ }^{18}$

\section{Results}

All the nine patients with DMD showed a reduction in the percentage of lymphocytes reaching the capping stage. The cap values observed in DMD ranged from 15 to 38 with a mean value of $31 \cdot 5 \pm$ 7.42 (table 2 ), while healthy controls presented cap percentages ranging from 44 to 61 with a mean value of $53 \cdot 39 \pm 4 \cdot 89$ (table 1 ).

Statistical evaluation of the capping percentage differences observed in healthy subjects and DMD patients, performed by the Mann-Whitney $U$ test, showed $p<0.00003$. The distribution of the capping capacities, detected by anti-HLA serum on the whole peripheral lymphocyte population in healthy subjects and DMD patients, is shown in the figure. In DMD patients cap capacities showed a negative correlation with CK levels $(\tau=-0.507, p=0.038)$ and a positive correlation with age $(\tau=+0.657$, $p=0.012$ ). Analysis of the data, performed by the Kendall partial rank correlation coefficient, confirmed the significant correlation between cap and age when $C K$ was partialled out $(\tau=+0 \cdot 604)$, while the correlation between cap and $\mathrm{CK}$ when age was held constant showed a reduction to $\tau=+0 \cdot 126$.

TABLE 2 Cell capping percentages, serum CK levels, and age in DMD patients.

\begin{tabular}{llcl}
\hline No & Capping (\%) & $C K(U / l)$ & Age $(y r)$ \\
\hline 1 & 38 & 322 & 20 \\
2 & 38 & 1137 & 10 \\
3 & 37 & 799 & 17 \\
4 & 35 & 1310 & 7 \\
5 & 35 & 1422 & 9 \\
6 & 30 & 1010 & 9 \\
7 & 29 & 1272 & 4 \\
8 & 27 & 1207 & 9 \\
9 & 15 & 1550 & 4 \\
\hline
\end{tabular}




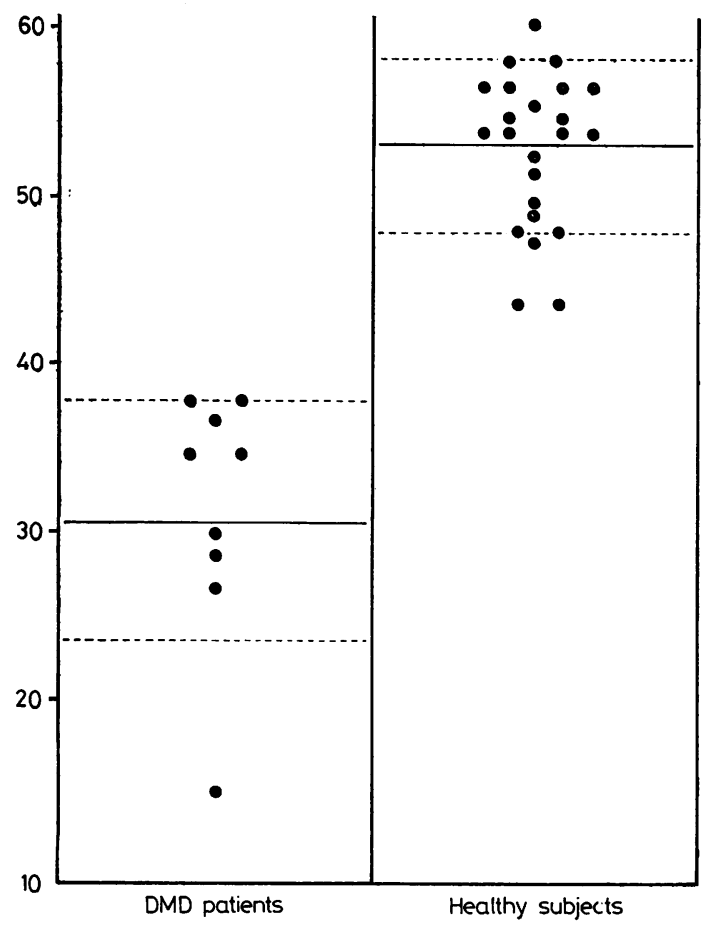

FIGURE HLA capping percentages in DMD patients and healthy subjects (mean values and standard deviations are shown).

However, no correlation was found in the 23 healthy subjects between cap capacity and age or CK level. Finally, cap percentages observed in neonates were not significantly correlated with $\mathrm{CK}$. levels $(\tau=-0 \cdot 352, \mathrm{p}=0 \cdot 130)$.

\section{Discussion}

Lymphocyte capping could be defined as an aggregation of the fluorescent antigen-antibody complexes into a single polar cap on the cell surface. A large number of factors, such as membrane structure and composition, availability of energy, and intracellular $\mathrm{Ca}^{++}$concentration are involved in the capping phenomenon. ${ }^{12-15}$

In the light of evidence of a systemic membrane abnormality in DMD patients, Verrill et $a^{1}$ and Pickard $e t a l^{2}$ reported impaired lymphocyte capping capacity in various muscular dystrophies. However, other investigators were unable to confirm the Ig capping reduction on B lymphocytes of DMD patients. ${ }^{4}$ 7-10

The evaluation of cell membrane function by the Ig capping capacity procedure, carried out on B lymphocytes, may be imprecise because of the low percentage of $\mathbf{B}$ cells in peripheral blood, which restricts the number of lymphocytes available for examination. In addition, because of the large number of cells not involved in the immunological reaction, some lymphocytes are non-specifically labelled, interfering with the determination of Ig induced capping.

In order to investigate the presence of a generalised membrane defect in DMD patients and to overcome technical difficulties in the evaluation of the capping phenomenon on B lymphocytes, we induced the cap formation of MHC molecules on the whole lymphocyte population.

The capping capacity of MHC molecules has been investigated in human cells for many years, and inefficient redistribution of these structures by direct HLA antisera treatment has been described.141519 Conjugated HLA antisera alone induced cap formation in only approximately $20 \% \overrightarrow{\text { }}$ of the total lymphocyte population, but the indirect immunofluorescent technique markedly increased $\bar{\nabla}$ the percentage of lymphocytes which reached the capping stage, raising it to 40 to $50 \%$.

The results obtained from our investigation on the MHC cap capacity in DMD patients extend the previous data of decreased capping in B cells to the whole lymphocyte population of peripheral blood.

The detection of reduced $T$ cell capacity is in agreement with Concanavalin A experiments described by Pickard et al, ${ }^{2}$ suggesting that the capping impairment in DMD patients does not arise from any specific characteristic of the surface Ig of $B$ lymphocytes, but is rather an expression of a generalised membrane defect involving the whole lymphocyte population.

The decreased Ig cap capacity in DMD patients seems related, on the basis of previous reports, to various factors such as age and CK serum level. ${ }^{6}$ However, conflicting results have been obtained in different studies and the meaning of such correlations is still uncertain. ${ }^{7}$

Our data, obtained on the whole lymphocyte population in DMD patients, show a positive correlation between cap capacity and age $(\tau=+0 \cdot 657$, $\mathrm{p}=0.012$ ), and a negative correlation between cap capacity and CK levels $(\tau=0.507, p=0.038)$. In addition, the analysis of our results, performed by the Kendall partial rank correlation coefficient, confirms the significance only for the correlation ? between cap and age when CK levels are partialled out ( $\left.\tau_{\text {cap/age, } \mathrm{CK}}=+0 \cdot 604\right)$.

These observations suggest that different 'factors', related to the activity of the disease rather than to the progression, are involved in the expression of the cap impairment. By this hypothesis, the gradual loss 
of muscular fibres in the progression of the dystrophy would produce smaller and smaller amounts of 'factors' affecting the degree of expression of the systemic membrane defect. Serum levels of CK are not strictly related to such unknown 'factors', since they are not linked to the degree of the capping impairment.

In conclusion, HLA capping performed on the whole lymphocyte population of peripheral blood seems to overcome some difficulties of Ig capping evaluation. Moreover, HLA capping shows no overlap between the ranges obtained in DMD patients and healthy controls, suggesting good results in the detection of the cellular impairment in DMD.

The normal range of the HLA capping capacities evaluated in the healthy newborn controls and the small amount of peripheral blood required could recommend our method as a diagnostic aid in the antenatal detection of hemizygous DMD males, unless the low disease activity in the fetus interferes with the detection of the systemic cellular impairment, because of the hypothesised relation with the cap capacity.

Furthermore, the results suggest that HLA investigation of DMD heterozygous females might be a method of carrier detection not related to $\mathrm{CK}$. serum levels.

\section{References}

1 Verrill HL, Pickard NA, Gruemer HD. Diminished cap formation in lymphocytes from patients and carriers of Duchenne muscular dystrophy. Clin Chem 1977;23: 2341-3.

2 Pickard NA, Gruemer HD, Verrill HL, et al. Systemic membrane defect in the proximal muscular dystrophies. N Engl J Med 1978;299:841-6.

3 Nash GB, Wyard SJ. Measurement of erythrocyte membrane elasticity as a diagnostic aid in Duchenne muscular dystrophy. J Med Genet 1982;19:262-5.

4 Fitzsimmons JS, McLachlan JI, Reeves WG, Marriott DW, Woolfson AMJ, Mayhew J. Carrier detection in Duchenne muscular dystrophy. J Med Genet 1980;17:156-69.
5 Hauser SL, Weiner HL, Bresnan MJ, Ault KA. Lymphocyte capping in muscular dystrophy. Neurology 1979;29: 1419-21.

6 Horenstein AL, Emery AEH. Human lymphocyte capping in Duchenne muscular dystrophy. Neurology 1980;30: 1330-2.

7 Nordal HJ, Andersson TR, Dietrichson P. Lymphocyte capping: a diagnostic method in progressive muscular dystrophy? Acta Neurol Scand 1982;65:442-7.

8 Sands ME, Harris R. Lymphocyte capping and carrier detection in Duchenne muscular dystrophy. Lancet 1979 ;ii:698.

- Stern CMM, Kahan MC, Dubowitz V. Lymphocyte capping in Duchenne muscular dystrophy. Lancet 1979; i: 1300.

${ }^{10}$ Sybert VP, Setran KN, Kadin ME. Lymphocyte capping in carriers of Duchenne muscular dystrophy. N EnglJ Med $1979 ; 301: 724-5$.

11 Singer SJ, Nicolson GL. The fluid mosaic model of the structure of cell membranes. Science 1972;175:720-31.

12 Emery AEH. Recent advances in the pathogenesis of Duchenne muscular dystrophy. J Med Genet 1982;19: 373A.

13 Verrill HL, Pickard NA, Gruemer HD. Mechanisms of cellular enzyme release. I. Alteration in membrane fluidity and permeability. Clin Chem 1977;23:2219-25.

14 Schreiner GF, Unanue ER. Capping and the lymphocyte: models for membrane reorganization. J Immunol 1977; 119:1549-51.

15 Schreiner GF, Unanue ER. Membrane and cytoplasmic changes in B lymphocytes induced by ligand-surface immunoglobulin interaction. Adv Immunol 1976;24: 37-150.

16 Bader PI. Lymphocyte capping of Duchenne muscular dystrophy. N Engl J Med 1979;301:725.

17 Rosalki SB. An improved method for creatine phosphokinase estimation. J Lab Clin Med 1967;69:696-705.

18 Siegel S. Nonparametric statistics for behavioral sciences. New York: McGraw-Hill, 1956.

19 Kourilsky FM, Silvestre D, Neuport-Sautes C, Loosfelt Y, Dausset J. Antibody-induced redistribution of HLA antigens at the cell surface. Eur J Immunol 1972;2 :249-57.

Correspondence and requests for reprints to Dr A Sensi, Istituto di Genetica Medica, Università degli Studi di Ferrara, Via L Borsari 46, 44100 Ferrara, Italy. 